Document downloaded from:

http://hdl.handle.net/10251/51424

This paper must be cited as:

Cantó Colomina, B.; Coll, C.; Sánchez, E. (2014). A study on vaccination models for a seasonal epidemic process. Applied Mathematics and Computation. 243:152-160. doi:10.1016/j.amc.2014.05.104.

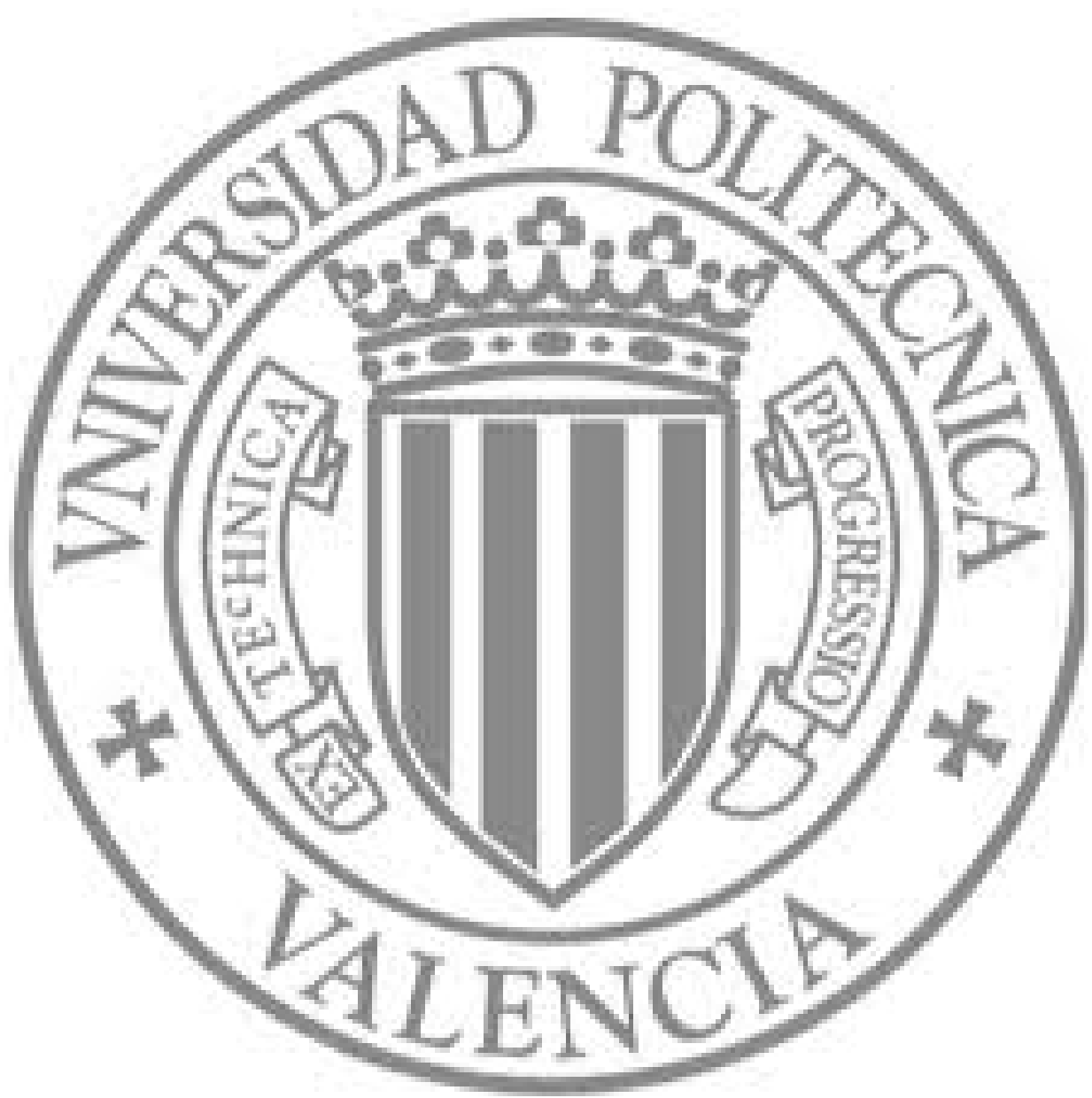

The final publication is available at

http://dx.doi.org/10.1016/j.amc.2014.05.104

Copyright Elsevier 


\title{
A study on vaccination models for a seasonal epidemic process
}

\author{
B. Cantó , C. Coll and E. Sánchez ${ }^{1}$ \\ Instituto de Matemática Multidisciplinar \\ Universitat Politècnica de València \\ Camino de Vera, 14. 46022 Valencia. Spain
}

\begin{abstract}
In this paper seasonal epidemiological processes are considered and a strategy of periodic vaccination is proposed. The invariant formulations associated with an $N$ - periodic system and the reproduction numbers associated with them are considered. New measures to study the stability of the system are introduced. Moreover, these new reproduction numbers help us to establish conditions on the periodic vaccination rates in the vaccination program. Finally, an SIR model is showed and a comparison between the results obtained using constant or periodic vaccination program is analyzed.
\end{abstract}

Keywords: epidemic process, seasonality, basic reproduction number, vaccination, discrete-time

93XX, 93A30, 93EXX

\section{Introduction}

The mathematical representation of an epidemiological process or a population growth process by means of a continuous-time or a discrete-time dynamic system has long been considered by many researchers, see $[1,2,3]$. In the study of these processes plays an important role the concept of basic reproduction number. This concept is defined as the spectral radius of a matrix or a linear operator constructed from the coefficient of the system, see $[4,5]$. The basic reproduction number is not just a measure or indicator to know whether the disease will be disappear altogether, but it is also a key to establish a threshold vaccination rate necessary to eradicate the disease, see for instance $[6,7]$.

Many epidemiological processes present seasonality. For example, rates of recovered individuals or of infected individuals may change with periodic behavior, according to the external conditions that affect the process. In this case, in the mathematical model appears periodic functions or matrices which hamper the analysis of the problem. The basic reproduction number associated with a

${ }^{1}$ Corresponding author. Email: esanchezj@mat.upv.es 
model with periodic coefficients of period $N$ has been defined by different authors, see $[8,9,10,11,12,13]$. In particular, in $[8,9]$ this concept is introduced as the spectral radius of a matrix which contains all the information for a period $N$ and in [13] the authors define $N$ reproduction numbers associated with the system.

The most common vaccination program is to vaccinate all individuals with a constant rate, see [8]. Perhaps it is the most direct method, but it is not the most effective. Some studies have considered specific cases of type SEIR or SEIRS continuous-time and they have done studies with pulse vaccination, see $[14,15]$.

Since it is important to take into account the periodicity of the process in the vaccination program, in this paper we consider seasonal epidemiological processes and we propose a strategy of periodic vaccination. Theory of linear dynamical systems in discrete-time and theory of nonnegative matrices and Mmatrices are the support of the theoretical development of this study. We introduce new measures or indicators to study the stability of the system. Finally, these new reproduction numbers help us to establish conditions on the periodic vaccination rates.

\section{Preliminaries and statement of work}

Consider an invariant system

$$
x(k+1)=E x(k), \quad E=T+F, \quad k \geq 0
$$

where the vector $x(k)$ represents the infected individuals at time $k$, the nonnegative matrices $T \geq O$ and $F \geq O$ are the transition matrix and the infection matrix, respectively.

The basic reproduction number, $\mathcal{R}_{0}$, quantifies the transmission potential of the disease and it gives information on transmissibility and contact rates. This indicator is defined by $\mathcal{R}_{0}=\rho\left(F(I-T)^{-1}\right)$, see [4]. Considering that the initial system without new infections is asymptotically stable $\rho(T)<1$, it is known (see [16]) that if $\mathcal{R}_{0}<1$ then the system (1) is asymptotically stable, that is, $\rho(E)=\rho(T+F)<1$. Moreover, if $\mathcal{R}_{0} \geq 1$, the system (1) is not asymptotically stable $\rho(E)=\rho(T+F) \geq 1$.

The basic reproduction number is used to find the threshold value of the vaccination rate, denoted by $v$, in order to obtain the eradication of the disease. If the matrix $F$ acts on the unvaccinated population then the vaccination model is given by

$$
x(k+1)=E^{v} x(k), \quad E^{v}=T+F(1-v), \quad k \geq 0 .
$$

Now, the new basic reproduction number is given by $\mathcal{R}_{0}^{v}=(1-v) \mathcal{R}_{0}$ and $1-\frac{1}{\mathcal{R}_{0}}$ is the threshold value for $v$ such that the model of vaccination becomes asymptotically stable $\rho\left(E^{v}\right)<1$, see [7]. That is, if $v>1-\frac{1}{\mathcal{R}_{0}}$ then $\mathcal{R}_{0}^{v}<1$.

When this procedure is applied to a seasonality process the difficulty of the problem increase. This seasonality leads to the periodicity of the coefficient 
matrices of the model. In this case the process is represented by an $N$-periodic system given by

$$
x(k+1)=E(k) x(k), \quad E(k)=T(k)+F(k), \quad k \geq 0,
$$

where the matrices are $N$-periodic matrices, that is, $T(k+N)=T(k) \geq O$, and $F(k+N)=F(k) \geq O, k \in \mathbb{Z}$.

In the literature, the $N$-periodic systems have been studied using the following two invariant formulations:

- $N$ IS: A collection of $N$ invariant systems (see [17]), given by

$$
x_{s}(k+1)=E_{s} x_{s}(k), \quad E_{s}=T_{s}+B_{s} F_{s}, \quad k \geq 0,
$$

where

$$
\begin{aligned}
& x_{s}(k)=x(k N+s), \quad B_{s}=\operatorname{row}\left[\Phi_{T}(s+N, s+1+j)\right]_{j=0}^{N-1}, \\
& T_{s}=\Phi_{T}(s+N, s) \quad F_{s}=\operatorname{col}\left[F(s+j) \Phi_{E}(s+j, s)\right]_{j=0}^{N-1}, \\
& \Phi_{T}\left(s, s_{0}\right)=\left\{\begin{array}{l}
\prod_{i=s_{0}}^{s-1} T\left(s_{0}+s-1-i\right), s>s_{0} \\
I_{n}, s=s_{0}
\end{array}\right.
\end{aligned}
$$

for $s=0,1, \ldots, N-1$.

- ICAS: An invariant system called Invariant Cyclically Augmented System, that recollect all the information of a period (see [18]) which state is

$$
\begin{aligned}
z(k)=M_{n}^{k-1} \widehat{x}(k), & \widehat{x}(k)=\operatorname{col}[x(k+j)]_{j=0}^{N-1} \\
M_{j} & =\left[\begin{array}{cc}
O & I_{(N-1) j} \\
I_{j} & O
\end{array}\right], j>0 .
\end{aligned}
$$

This invariant system is given by

$$
z(k+1)=E_{e} z(k), \quad E_{e}=T_{e}+F_{e}, \quad k \geq 0
$$

where $T_{e}$ and $F_{e}$ have the following structure

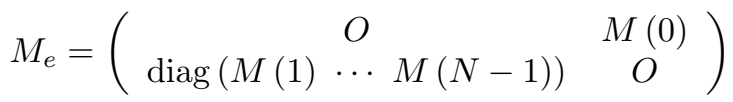

using the corresponding $N$-periodic collection of matrices.

It is known that the $N$-periodic system is asymptotically stable if and only $\rho\left(T_{s}\right)<1$ or equivalently $\rho\left(T_{e}^{N}\right)<1$, since $T_{e}^{N}=\operatorname{diag}\left(T_{1} T_{2} \cdots T_{n-1} T_{0}\right)$.

Given an $N$-periodic system, a first approach of concept of basic reproduction number leads us to define this measure as the basic reproduction number of its ICAS, that is, $\mathcal{R}_{0}^{e}=\rho\left(F_{e}\left(I-T_{e}\right)^{-1}\right)$, see $[8,9]$. Another way is to define it using its NIS formulation, that is, $\mathcal{R}_{0}^{s}=\rho\left(B_{s} F_{s}\left(I-T_{s}\right)^{-1}\right), s=0,1, \ldots N-1$, see [13]. 
In order to get the eradication of the disease we can plan different ways of actuation. If we consider a constant vaccination program, that is, if we consider in all seasons the infection matrix at time $k$ equal to $(1-v) F(k)$, for all $k=0,1, \ldots, N-1$, the vaccination model is given by

$$
x(k+1)=E^{v}(k) x(k), \quad E^{v}(k)=T(k)+(1-v) F(k), \quad k \geq 0 .
$$

In this case, the new reproduction number using the ICAS formulation is $\mathcal{R}_{0}^{e v}=$ $\rho\left(F_{e}^{v}\left(I-T_{e}\right)^{-1}\right)=(1-v) \mathcal{R}_{0}^{e}$ since $F_{e}^{v}=(1-v) F_{e}$. Then, the threshold value for $v$ such that the model of vaccination becomes asymptotically stable is $1-\frac{1}{\mathcal{R}_{0}^{e}}$, see [9].

If we consider the NIS formulation, then the reproduction numbers are $\mathcal{R}_{0}^{s v}=\rho\left(B_{s} F_{s}^{v}\left(I-T_{s}\right)^{-1}\right), s=0,1, \ldots N-1$, being

$$
F_{s}^{v}=\operatorname{col}\left[F^{v}(s) \Phi_{E^{v}}(s+j-1, s)\right]_{j=1}^{N}
$$

It is not straightforward to obtain a relation between $\mathcal{R}_{0}^{s v}$ and $\mathcal{R}_{0}^{s}, s=0,1, \ldots N-$ 1.

Note that considering $v$ at all time we are forgetting the seasonality of the process. It seems logical to think that the vaccinated population rate in each stage or season must be related to the changing conditions affecting that season. That is, the periodic variation of the characteristics of the process should influence the rate of population to be vaccinated at each step. Thus, if we consider the rate $v_{s}$ vaccination in step $s, s=0,1, \ldots, N-1$, the model is given by

$$
x(k+1)=E^{v}(k) x(k), \quad E^{v}(k)=T(k)+\left(1-v_{k}\right) F(k), \quad k \geq 0 .
$$

Now, the ICAS formulation does not provide a direct way to find the threshold values of $v_{s}, s=0,1, \ldots, N-1$. We focus our attention on this problem. Our goal is to find conditions on the collection in order to improve the threshold value obtained when the rate of vaccinated population is the same at all steps. For this, in the Section 3 we introduce new measures or indicators associated with the $N$-periodic system and we study the stability of the system from these indicators. In Section 4, we give conditions on the collection of vaccination rates $v_{s}, s=0,1, \ldots, N-1$ in order to the vaccination model becomes asymptotically stable. Finally, in the Section 5, we consider an SIR model and we compare between the results obtained using constant or periodic vaccination strategy.

\section{Reduced reproduction numbers.}

Consider the $\mathrm{N}$-periodic system given in (2) and the coefficient matrices of the ICAS and NIS formulations. Then, we establish the following result which proof is straightforward using the definition of the involved matrices.

\section{Proposition 1.}

(i) For $j=0, \ldots, N-1$

$$
T_{e}^{j} F_{e} E_{e}^{j}=\operatorname{diag}\left(\Phi_{T}(s+N, s+1+j) F(s+j) \Phi_{E}(s+j, s)\right)_{s=1}^{N} .
$$


(ii) Denoting $\bar{B}_{e}=\left(T_{e}^{N} T_{e}^{N-1} \cdots I\right)$ and $\bar{F}_{e}=\operatorname{col}\left[F_{e} E_{e}^{j}\right]_{j=0}^{N-1}$. we have

$$
\operatorname{diag}\left(B_{s} F_{s}\left(I-T_{s}\right)^{-1}\right)_{s=1}^{N}=\bar{B}_{e} \bar{F}_{e}\left(I-T_{e}^{N}\right)^{-1} .
$$

Next, we define the new collection of measures, which will help us in the study of a threshold property associated with the stability of the system.

Definition 1. For each $j, j=0,1, \ldots, N-1$, we define

(i) the s-basic reproduction number at time $j$ as $\mathcal{R}_{j}(s)=\rho\left(M_{j}(s)\right), s=$ $0,1, \ldots, N-1$ with

$$
\begin{aligned}
& M_{j}(s)=Y_{j}(s)\left(I-X_{j}(s)\right)^{-1}, \text { where } \\
& X_{j}(s)=T_{s}+\sum_{i=0}^{j-1} Y_{i}(s) \text { if } j=1, \ldots, N-1, \text { and } X_{0}(s)=T_{s}, \\
& Y_{j}(s)=\Phi_{T}(s+N, s+1+j) F(s+j) \Phi_{E}(s+j, s) .
\end{aligned}
$$

And, from the periodicity of matrices of the system, $\mathcal{R}_{j}(k+N)=\mathcal{R}_{j}(k)$, $k \in \mathbb{Z}$.

(ii) the reduced reproduction number at time $j$ of the system (2) as

$$
\begin{aligned}
& \mathcal{R}_{j}^{e}=\rho\left(M_{j}^{e}\right)=\max \left\{\mathcal{R}_{j}(s), s=0,1, \ldots, N-1\right\} \\
& \text { being } M_{j}^{e}=T_{e}^{j} F_{e} E_{e}^{j}\left(I-X_{e}^{j}\right)^{-1} \text { with } X_{e}^{j}=T_{e}+\sum_{i=0}^{j-1} T_{e}^{i} F_{e} E_{e}^{i}, \text { if } j= \\
& 1, \ldots, N-1 \text {, and } X_{e}^{0}=T_{e} .
\end{aligned}
$$

Since the eradication of the disease is obtained when the system is asymptotically stable, in the following result we give conditions on the above collection of reproduction numbers $\left\{\mathcal{R}_{j}^{e}, j=0,1, \ldots, N-1\right\}$ in order to get the stability of the system.

\section{Proposition 2.}

(i) Let $j$ be such that $\mathcal{R}_{j}^{e}<1$. If $\rho\left(X_{j}(s)\right)<1$ then $\rho\left(X_{j}(s)+Y_{j}(s)\right)<1$.

(ii) If $\mathcal{R}_{N-1}^{e}<1$ and $\rho\left(X_{N-1}(s)\right)<1$, then the $N$-periodic system (2) is asymptotically stable.

(iii) If there exists $j$ and $s$ such that $\mathcal{R}_{j}(s) \geq 1$ and $\rho\left(X_{j}(s)\right)<1$ then the $N$-periodic system (2) is not asymptotically stable.

\section{Proof.}

(i) Suppose that $\mathcal{R}_{j}^{e}<1$, then $\mathcal{R}_{j}(s)=\rho\left(M_{j}(s)\right)<1$ for all $s=0,1, \ldots, N-1$. From $\rho\left(X_{j}(s)\right)<1$, taking into account $I-M_{j}(s)$ is an M-matrix nonsingular and $I-M_{j}(s)=I-Y_{j}(s)\left(I-X_{j}(s)\right)^{-1}=\left(I-X_{j}(s)-Y_{j}(s)\right)\left(I-X_{j}(s)\right)^{-1}$, then $I-X_{j}(s)-Y_{j}(s)$ is nonsingular and its inverse matrix is nonnegative

$$
\left(I-X_{j}(s)-Y_{j}(s)\right)^{-1}=\left(I-X_{j}(s)\right)^{-1}\left(I-M_{j}(s)\right)^{-1} \geq O .
$$


Thus, we can ensure that $I-\left(X_{j}(s)+Y_{j}(s)\right)$ is an M-matrix nonsingular. Hence, $\rho\left(X_{j}(s)+Y_{j}(s)\right)<1$.

(ii) In the above result when $j=N-1$ we have $X_{N-1}(s)+Y_{N-1}(s)=T_{s}+B_{s} F_{s}$, then $\rho\left(T_{s}+B_{s} F_{s}\right)<1$ and the $N$-periodic system (2) is asymptotically stable.

(iii) If there exists $j$ and $s$ such that $\mathcal{R}_{j}(s) \geq 1$, then $\rho\left(X_{j}(s)+Y_{j}(s)\right) \geq 1$.

In order to know if the $N$-periodic system is stable or unstable, we use its $N$ IS formulation where $E_{s}=T_{s}+B_{s} F_{s} \geq O$. By construction of matrices $X_{j}(s)$ and $Y_{j}(s)$, we have $E_{s}=T_{s}+B_{s} F_{s} \geq X_{j}(s)+Y_{j}(s)$, and so, $\rho\left(E_{s}\right) \geq$ $\rho\left(X_{j}(s)+Y_{j}(s)\right) \geq 1$, since they are nonnegative matrices. Then, the $N$-periodic system is not asymptotically stable and the proof is finished.

To clarify the above concepts we show the different reproduction numbers associated with a 2-periodic system given by $E(k)=A(k)+F(k), E(k+2)=$ $E(k), k \in \mathbb{Z}$. By definition of the ICAS formulation and NIS formulation we have

$$
\begin{aligned}
& \mathcal{R}_{0}^{e}=\rho\left(F_{e}\left(I-T_{e}\right)^{-1}\right)=\rho\left(\left(\begin{array}{cc}
O & F(0) \\
F(1) & O
\end{array}\right)\left(\begin{array}{cc}
I & -T(0) \\
-T(1) & I
\end{array}\right)^{-1}\right) \\
& \mathcal{R}_{0}^{0}=\rho\left(B_{0} F_{0}\left(I-T_{0}\right)^{-1}\right)=\rho\left((T(1) F(0)+F(1) E(0))\left(I-T_{0}\right)^{-1}\right) \\
& \mathcal{R}_{0}^{1}=\rho\left(B_{1} F_{1}\left(I-T_{1}\right)^{-1}\right)=\rho\left((T(0) F(1)+F(0) E(1))\left(I-T_{1}\right)^{-1}\right)
\end{aligned}
$$

and by Definition 1 we have the new measures

$$
\begin{array}{ll}
j=0 & \mathcal{R}_{0}(0)=\rho\left(M_{0}(0)\right)=\rho\left(T(1) F(0)\left(I-T_{0}\right)^{-1}\right) \\
& \mathcal{R}_{0}(1)=\rho\left(M_{0}(1)\right)=\rho\left(T(0) F(1)\left(I-T_{1}\right)^{-1}\right) \\
j=1 & \mathcal{R}_{1}(0)=\rho\left(M_{1}(0)\right)=\rho\left(F(1) E(0)\left(I-T_{0}-T(1) F(0)\right)^{-1}\right) \\
& \mathcal{R}_{1}(1)=\rho\left(M_{1}(1)\right)=\rho\left(F(0) E(1)\left(I-T_{1}-T(0) F(1)\right)^{-1}\right)
\end{array}
$$

\section{Vaccination models}

We are going to discuss the basic reproduction number and the impact on the vaccination programs in the infectious diseases. In this section, the focus is in the dynamic changes and we will analyze the critical vaccination proportion needed to eradicate the disease.

As we have already mentioned in Section 2, in a seasonal epidemiologic process, the usual vaccination program so far has been based on the use of ICAS formulation. Thus, it has been used a constant rate of vaccination, $v$, the same in all steps. The threshold value of $v$ such that the model becomes asymptotically stable vaccination is $1-\frac{1}{\mathcal{R}_{0}^{e}}$.

Our proposal is to achieve the eradication of disease using vaccination such that the rate of the vaccinated population depends on each step or season. For that, we consider the new infection matrix at time $s$ is $F^{v}(s)=\left(1-v_{s}\right) F$, for every $s=0,1, \ldots, N-1$, and the vaccination model is represented by the system given in (3). To find the threshold values of the rates of $\left\{v_{s}, 0 \leq v_{s} \leq\right.$ $1, s=0,1, \ldots, N-1\}$ analyze the stability measures introduced in the previous section. For each $j, j=0,1, \ldots, N-1$, the $s$-basic reproduction numbers at time 
$j$ of the vaccinated model are going to be $\mathcal{R}_{j}^{v}(s)=\rho\left(M_{j}^{v}(s)\right), s=0,1, \ldots, N-1$ with

$$
\begin{aligned}
& M_{j}^{v}(s)=Y_{j}^{v}(s)\left(I-X_{j}^{v}(s)\right)^{-1}, \text { where } \\
& X_{j}^{v}(s)=T_{s}+\sum_{i=0}^{j-1} Y_{i}^{v}(s) \text { if } j=1, \ldots, N-1, \text { and } X_{0}^{v}(s)=T_{s}, \\
& Y_{j}^{v}(s)=\Phi_{T}(s+N, s+1+j) F^{v}(s+j) \Phi_{E^{v}}(s+j, s) .
\end{aligned}
$$

And, the reduced reproduction number at time $j$ of the vaccinated model is

$$
\mathcal{R}_{j}^{e v}=\max \left\{\mathcal{R}_{j}^{v}(s), s=0,1, \ldots, N-1\right\} .
$$

In the following result we give conditions on a collection of values $\left\{v_{s}, s=\right.$ $0,1, \ldots, N-1\}$ in order to get eradicate the disease in a seasonal model.

Proposition 3. Consider a not asymptotically stable $N$-periodic system given by (2). If there exists a collection $\left\{v_{s}, 0 \leq v_{s} \leq 1, s=0,1, \ldots, N-1\right\}$ satisfying

$$
1-v_{s+j}<\frac{1}{\widetilde{\mathcal{R}}_{j}^{v}(s)}, 0<j \leq N-1,
$$

with $\widetilde{\mathcal{R}}_{j}^{v}(s)=\rho\left(\Phi_{T}(s+N, s+1+j) F \Phi_{E^{v}}(s+j, s)\left(I-X_{j}^{v}(s)\right)^{-1}\right)$, then, the vaccination model given by (3) is asymptotically stable.

Proof. Since system (2) is not asymptotically stable, $\rho\left(E_{e}^{N}\right) \geq 1$, then, for some $j, \mathcal{R}_{j}^{e} \geq 1$. We suppose $j_{0}$ such that $\mathcal{R}_{j}^{e}<1$ for all $j<j_{0}$ and $\mathcal{R}_{j_{0}}^{e} \geq 1$. By definition of $\mathcal{R}_{j_{0}}^{e}$, there exists almost $s$ such that $\mathcal{R}_{j_{0}}(s) \geq 1$. Without loss of generality we consider $j_{0}=0$. Then, we consider a periodic vaccination program such that

$$
\mathcal{R}_{0}^{v}(s)=\left(1-v_{s}\right) \mathcal{R}_{0}(s)<1, s=0,1, \ldots, N-1 .
$$

Thus, if the collection $\left\{v_{s}, 0 \leq v_{s} \leq 1, s=0,1, \ldots, N-1\right\}$ satisfies the above condition, then $\mathcal{R}_{0}^{e v}<1$. Moreover, to control the disease it is necessary that $\mathcal{R}_{j}^{e v}<1$, for all $0 \leq j \leq N-1$. Hence

$$
\begin{aligned}
& \mathcal{R}_{j}^{v}(s)=\rho\left(Y_{j}^{v}(s)\left(I-X_{j}^{v}(s)\right)^{-1}\right)= \\
& =\rho\left(\Phi_{T}(s+N, s+1+j) F^{v}(s+j) \Phi_{E^{v}}(s+j, s)\left(I-X_{j}^{v}(s)\right)^{-1}\right)=\left(1-v_{s+j}\right) \widetilde{\mathcal{R}}_{j}^{v}(s) .
\end{aligned}
$$

Then, the conditions

$$
1-v_{s+j}<\frac{1}{\widetilde{\mathcal{R}}_{j}^{v}(s)}, j_{0}<j \leq N-1,
$$

established in the statement are hold.

Note that from the periodicity, the above conditions are equivalent to the collection $\left\{v_{s}, 0 \leq v_{s} \leq 1, s=0,1, \ldots, N-1\right\}$ satisfies the following condition

$$
1-v_{s}<\left\{\frac{1}{\widetilde{\mathcal{R}}_{0}^{v}(s)}, \frac{1}{\widetilde{\mathcal{R}}_{1}^{v}(s-1)}, \ldots, \frac{1}{\widetilde{\mathcal{R}}_{N-1}^{v}(s-(N-1))}\right\} .
$$


When $N=2$ the above conditions are

$$
\begin{aligned}
& 1-v_{0}<\left\{\frac{1}{\widetilde{\mathcal{R}}_{0}^{v}(0)}, \frac{1}{\widetilde{\mathcal{R}}_{1}^{v}(1)}\right\} \\
& 1-v_{1}<\left\{\frac{1}{\widetilde{\mathcal{R}}_{0}^{v}(1)}, \frac{1}{\widetilde{\mathcal{R}}_{1}^{v}(0)},\right\}
\end{aligned}
$$

where

$$
\begin{array}{ll}
j=0 & \widetilde{\mathcal{R}}_{0}^{v}(0)=\rho\left(T(1) F\left(I-T_{0}\right)^{-1}\right)=\mathcal{R}_{0}(0) \\
& \widetilde{\mathcal{R}}_{0}^{v}(1)=\rho\left(T(0) F\left(I-T_{1}\right)^{-1}\right)=\mathcal{R}_{0}(1) \\
j=1 & \widetilde{\mathcal{R}}_{1}^{v}(0)=\rho\left(F\left(E^{v}(0)\left(I-T_{0}-T(1) F\left(1-v_{0}\right)\right)^{-1}\right)\right. \\
& \widetilde{\mathcal{R}}_{1}^{v}(1)=\rho\left(F E^{v}(1)\left(I-T_{1}-T(0) F\left(1-v_{1}\right)\right)^{-1}\right)
\end{array}
$$

with $E^{v}(s)=T(s)+\left(1-v_{s}\right) F, s=0,1$.

In the following section we introduce a seasonality SIR model and we study the vaccination programs comparing between the obtained results, using the same or different rate of vaccination at all season.

\section{Application to a seasonality SIR model}

The SIR model is one of the basic compartmental models in infections disease epidemiology, which is widely used and well suited to model many viral infectious in childhood. Let us consider a transmission model consisting of three compartments: susceptible $(S)$, infected $(I)$, and immune or recovered $(R)$. An underlying assumption of this $S I R$ model is that individuals are born into the susceptible class. After infection, the individuals transfer to the infected class and after clearing the infection individuals are transferred to the recovered class. It is assumed that, after recovery, individuals gain lifelong immunity and therefore do not take part in the transmission process other than that they represent the complement of those that do (i.e., the more immune people, the fewer there can be infectious and susceptible people in the population).

If $\beta$ denotes the births added to $S$ state and $p, q, \alpha$ and $r$ are the survival rate of the $S, I, S \cdot I$ and $R$ individuals, respectively, and $\gamma(k)$ denotes the rate of infectious individual becoming removed individual, we can modelled the SIR model using the following discrete-time system

$$
\begin{aligned}
S(k+1) & =p S(k)-\alpha S(k) I(k)+\beta N(k) \\
I(k+1) & =q I(k)+\alpha S(k) I(k)-\gamma(k) I(k) \\
R(k+1) & =r R(k)+\gamma(k) I(k), \quad N(k)=S(k)+I(k)+R(k),
\end{aligned}
$$

Note that $\gamma(k+N)=\gamma(k)$, with $N=2$, is a periodical varying parameter in this model. This means that we have considered seasonality in the removed individuals. 
This model is more realistic because usually some diseases are more easily recovered in summer that in winter, in dependence of the weather.

Then, we linearize around the disease-free equilibrium point obtaining the following linear system

$$
\begin{aligned}
& S(k+1)=p S(k)-h I(k)+\beta N(k) \\
& I(k+1)=(q(1-\gamma(k))+h) I(k) \\
& R(k+1)=q \gamma(k) I(k)+r R(k)
\end{aligned}
$$

where $h$ is the rate of susceptible individuals becoming infectious individual.

The evolution of the disease state is given by infected individuals, and hence we can reduce this model to the infected linear model. Moreover, we consider an example where the influence of the diseases differs according to the case of juvenile individuals or adults individuals. Then, the infected population is divided into two classes: $I(k)=\left(I_{j}(k) I_{a}(k)\right)^{T}$. Denoting

- $h_{j} / h_{a}$ the rate of individuals becoming infectious juvenile/adult individuals,

- $q_{j} / q_{a}$ the survival rate of the class $I_{j} / I_{a}$,

- $\gamma_{j}(k) / \gamma_{a}(k)$ the rate of infectious individual becoming removed individual at time $k, k=0,1$,

- $s$ the rate of infected juvenile individuals becoming infected adult individuals.

we have the following 2-periodic system

$$
\begin{aligned}
& I(k+1)=(T(k)+F) I(k)= \\
& \quad=\left(\left(\begin{array}{cc}
q_{j}\left(1-\gamma_{j}(k)-s\right) & 0 \\
q_{j} s & q_{a}\left(1-\gamma_{a}(k)\right)
\end{array}\right)+\left(\begin{array}{cc}
h_{j} & 0 \\
0 & h_{a}
\end{array}\right)\right) I(k)
\end{aligned}
$$

In order to apply control to this problem, firstly we consider the vaccination of the population at a fixed rate $v$. If a proportion $v$ of the individuals is vaccinated successfully, then these individuals are assumed to become immune and they are removed from the original class. These assumptions lead to the following equation:

$$
I(k+1)=(T(k)+(1-v) F) I(k) .
$$

If $\mathcal{R}_{0}^{e}<1$ no vaccination is required in order to maintain a disease-free population, while for $\mathcal{R}_{0}^{e} \geq 1$, we need that the vaccination rate, $v$, satisfies $1-v<\frac{1}{\mathcal{R}_{0}^{e}}$ to ensure that $\mathcal{R}_{0}^{e v}<1$. Likewise, we analyze the stability using $N$ IS formulation and the reproduction numbers $\mathcal{R}_{0}^{0}$ and $\mathcal{R}_{0}^{1}$.

The idea of using constant vaccination rate is not very realistic. In practice, population groups are vaccinated during very short time intervals for logistic reasons. This means that impulse control is better control model than the constant vaccination rate model. Then, when the population have been vaccinated 
in this way, the new matrices $F^{v}(s)$ depend on the rate of the vaccination, $v_{0}$ and $v_{1}$. Thus, $F^{v}(0)=F\left(1-v_{0}\right)$ and $F^{v}(1)=F\left(1-v_{1}\right)$. In this case, by means of the reproduction numbers associated with the ICAS or NIS formulation it is difficult to obtain the threshold values to $v_{0}$ and $v_{1}$. This motivates the use of the $s$-basic reproduction number at time $j, \mathcal{R}_{j}(s)=\rho\left(M_{j}(s)\right), s=0,1$, $j=0,1$.

In the following example we apply the proposed approach and we compare it with the case where it is considered the constant rate of vaccination.

Example 1. We consider the following dates:

$$
\begin{aligned}
& q_{j}=0.9, q_{a}=0.7, h_{j}=0.7, h_{a}=0.3, s=0.1 \\
& \gamma_{j}(0)=0.1, \gamma_{a}(0)=0.5, \gamma_{j}(1)=0.5, \gamma_{a}(1)=0.8
\end{aligned}
$$

Then, the 2-periodic system is not asymptotically stable, since $\rho\left(E_{s}\right)=1.5$. Moreover the reproduction number associated with the ICAS and NIS formulations are greater than 1:

$$
\begin{aligned}
& \mathcal{R}_{0}^{e}=\rho\left(B_{e} F_{e}\left(I-T_{e}\right)^{-1}\right)=1.47 \\
& \mathcal{R}_{0}^{0}=\rho\left(B_{0} F_{0}\left(I-T_{0}\right)^{-1}\right)=1.68=\mathcal{R}_{0}^{1}=\rho\left(B_{1} F_{1}\left(I-T_{1}\right)^{-1}\right) .
\end{aligned}
$$

By Definition 1 we have the new measures

$$
\begin{array}{ll}
j=0 & \mathcal{R}_{0}(0)=0.34 \\
& \mathcal{R}_{0}(1)=0.68 \\
j=1 & \mathcal{R}_{1}(0)=2.03 \\
& \mathcal{R}_{1}(1)=3.13 .
\end{array}
$$

Hence, the reduced reproduction number at time 0 is $\mathcal{R}_{0}^{e}=0.68$ and at time 1 is $\mathcal{R}_{1}^{e}=3.13 \geq 1$.

By definitions of $\widetilde{\mathcal{R}}_{j}^{v}(s)$ given in (5) we have

$$
\begin{array}{ll}
j=0 & \widetilde{\mathcal{R}}_{0}^{v}(0)=\mathcal{R}_{0}(0)=0.34 \\
& \widetilde{\mathcal{R}}_{0}^{v}(1)=\mathcal{R}_{0}(1)=0.68 \\
j=1 & \widetilde{\mathcal{R}}_{1}^{v}(0)=\frac{0.7\left(0.72+0.7\left(1-v_{0}\right)\right)\left(0.951-0.042\left(1-v_{0}\right)\right)}{0.44+0.25 v_{0}+0.01 v_{0}^{2}} \\
& \widetilde{\mathcal{R}}_{1}^{v}(1)=\frac{0.7\left(0.36+0.7\left(1-v_{1}\right)\right)\left(0.95-0.1\left(1-v_{1}\right)\right)}{0.2+0.45 v_{1}+0.05 v_{1}^{2}},
\end{array}
$$

and from Proposition 3 and the conditions given in (4) we have that $\left\{v_{0}, v_{1}\right\}$ have to satisfy

$$
\begin{aligned}
& 1-v_{0}<\left\{\frac{1}{0.34}, \frac{0.2+0.45 v_{1}+0.05 v_{1}^{2}}{0.7\left(0.36+0.7\left(1-v_{1}\right)\right)\left(0.95-0.1\left(1-v_{1}\right)\right)}\right\} \\
& 1-v_{1}<\left\{\frac{1}{0.68}, \frac{0.44+0.24 v_{0}+0.01 v_{0}^{2}}{0.7\left(0.72+0.7\left(1-v_{0}\right)\right)\left(0.951-0.042\left(1-v_{0}\right)\right)}\right\} .
\end{aligned}
$$


On the other hand, if we consider constant rate of vaccination $v$ and consider the ICAS formulation

$$
\mathcal{R}_{0}^{e v}=\rho\left(F_{e}^{v}\left(I-T_{e}\right)^{-1}\right)=(1-v) \mathcal{R}_{0}^{e}=1.47(1-v),
$$

with $F_{e}^{v}=(1-v) F_{e}$. The threshold value for $v$ such that the model of vaccination becomes asymptotically stable is $1-\frac{1}{\mathcal{R}_{0}^{e}}=0.32$.

The evolution of the disease is measure from the spectral radius of the matrix $E_{e}^{v}=T_{e}+F_{e}^{v}$ of ICAS formulation or $E_{s}^{v}=T_{s}+F_{s}^{v}$ of NIS formulation of the vaccination model.

In this example, it is necessary a higher level of vaccination in the constant vaccination program than in the periodic vaccination program to obtain a similar evolution in the dynamics of the infected population from constant or periodic vaccination strategy. For example, for the vaccination model to be asymptotically stable with $\rho\left(E_{s}^{v}\right)=0.81<1$, in the constant strategy we have to vaccinate $46 \%$ of the population during the two steps while in the periodic vaccination strategy will only have to vaccinate $70 \%$ of the population in one of the steps. Moreover, a constant vaccination equal to $35 \%$ leads to $\rho\left(E_{s}^{v}\right)=0.95<1$. In this case the eradication of the disease is slower than vaccinating only at odd steps with $70 \%$.

In the NIS formulation of the infected model, the states are given by $I_{s}^{j}(k)=$ $I_{j}(k N+s)$ and $I_{s}^{a}(k)=I_{a}(k N+s)$ and they represent the juvenile/adult infected population at time $k N+s$.

In the Figure 1 we show the evolution of the juvenile/adult infected population before of the vaccination and after of a periodic vaccination program. And, the Figure 2 show this evolution after of two different constant vaccination programs.

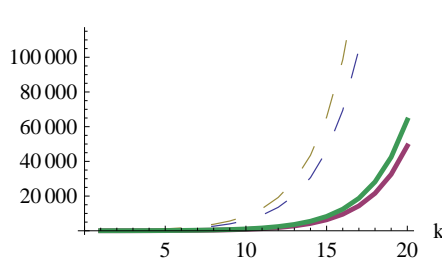

(a) Without vaccination

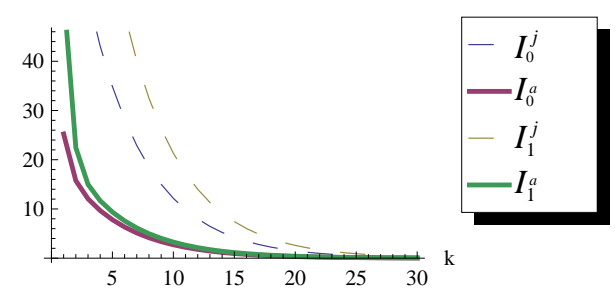

(b) Periodic vaccination $v_{0}=0, v_{1}=0.7$

Figure 1: Evolution of infected individuals $I_{s}^{j}(k)$ and $I_{s}^{a}(k):(a)$ Before of any vaccination program. (b) After of the periodic vaccination program, $v_{0}=0$ and $v_{1}=0.7$. 


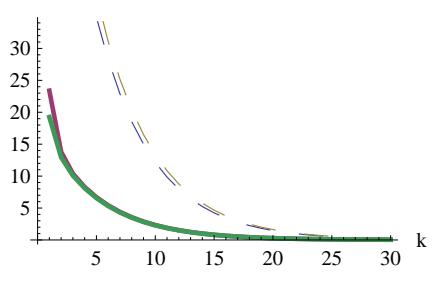

(a) Constant vaccination $v=0.46$

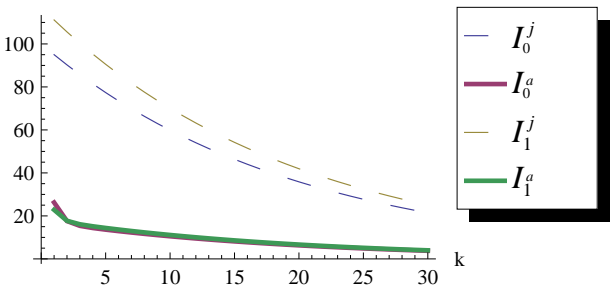

(b) Constant vaccination $v=0.35$

Figure 2: Evolution of infected individuals $I_{s}^{j}(k)$ and $I_{s}^{a}(k):$ (a) After of the constant vaccination program, $v=0.46$. (b) After of the constant vaccination program, $v=0.35$.

\section{Conclusions}

Seasonal epidemiological processes represented by $N$-periodic systems are considered join with its ICAS and NIS formulations. Different basic reproduction number approaches are introduced to analyze the eradication of the disease. Using theory of linear dynamical systems in discrete-time and theory of nonnegative matrices and M- matrices a property about the asymptotic stability of the system is established. Motivate by the importance of the periodic property of the process a periodic vaccination program is studied. Conditions on the vaccination rates in this program are given in terms of the new basic reproduction numbers.

The results presented herein are applied to a seasonal epidemiological model with two compartments in the infected individuals. This analysis is focus on a comparison between the behaviour of the infected population using constant or periodic vaccination program. A higher level of vaccination in the constant vaccination program than in the periodic vaccination program is needed to obtain a similar evolution in the dynamics of the infected population.

\section{References}

[1] H. Cao, Y. Zhou, The discrete age-structured SEIT model with application to tuberculosis transmission in China, Mathematical and Computer Modelling (2012), 385-395.

[2] P. van den Driessche, J. Watmough, Reproduction numbers and subthreshold endemic equilibria for compartmental models of disease transmission, Mathematical Biosciences 180 (2002), 29-48.

[3] X. Li, W. Wang, A discrete epidemic model with stage structure, Chaos Solitons \& Fractals 26 (2006), 947-958.

[4] L. Allen, P. van den Driessche, The basic reproduction number in some discrete-time epidemic models, J. Difference Equ. Appl. (2008), 1-19. 
[5] O. Diekmann, J.A.P. Heesterbeek, J.A.J. Metz, On the definition and the computation of the basic reproduction ratio $R_{0}$ in models for infectious diseases in heterogeneous populations, Journal Math. Biol. 28 (1990), pp. $365-382$.

[6] C.P. Farrington, On vaccinate efficacy and reproduction numbers, Mathematical Biosciences 185 (2003), 89-109.

[7] A. Scherer, A. McLean, Mathematical models of vaccination, British Medical Bulletin 62 (2002), 187-199.

[8] N. Bacaër, Periodic matrix population models: growth rate, basic reproduction number; and entropy, Bulletin Math. Biol. 71 (2009), 1781-1792.

[9] N. Bacaër, E.H.A. Dads, On the biological interpretation of a definition for the parameter $R_{0}$ in periodic population models, Journal Mathematical Biology (2012), 601-621.

[10] H. Cao, Y. Zhou, The basic reproduction number of discrete SIR and SEIS models with periodic parameters, Discrete and Continuous dynamical Systems Series B 18(1) (2013), 37-56.

[11] Y. Nakata, T. Kuniya, Global Dynamics of a class of SEIRS epidemic models in a periodic environment, Journal of Mathematical Analysis and Applications 363 (2010), 230-237.

[12] W. Wang, X.Q. Zhao, Threshold Dynamics for Compartmental Epidemic Models in Periodic Environments, Journal Dynamic Differential Equations 20 (2008), 699-717.

[13] J.M. Cushing, A.S. Ackleh, A net reproductive number for periodic matrix models. J. Biol. Dyn. 6(2) (2012), 166-188.

[14] M de la Sen, S. Alonso-Quesada, A. Ibeas, On the Equilibrium Point and some Properties of a SVEIRS Epidemic Model, in: Procc. of the World Congress on Engineering, London, 2011.

[15] A. d'Onofrio, Mixed pulse vaccination strategy in epidemic model with realistically distributed infections and latent times, Applied Mathematics and Computation 151 (2004), 181-187.

[16] C.K. Li, H. Schneider, Applications of Perron-Frobenius theory to population dynamics, J. Math. Biol. 44 (2002), 450-462.

[17] R.A. Meyer, C.S. Burrus, A unified analysis of multirate and periodically time-varying digital filters, IEEE Trans. Circuits Systems 22(3) (1975), $162-168$.

[18] B. Park, E.I. Verriest, Canonical forms on discrete linear periodically timevarying system and a control application, in: Procc. 28th IEEE Conf. on Decision and Control, Tampa, 1989, pp. 1220-1225. 
E-mail address: bcanto@mat.upv.es

E-mail address: mccoll@mat.upv.es

E-mail address: esanchezj@mat.upv.es 